\title{
Biologia reprodutiva de Cercosaura schreibersii (Squamata, Gymnophthalmidae) e Cnemidophorus lacertoides (Squamata, Teiidae) no Escudo Sul-Riograndense, Brasil
}

\author{
Rafael Lucchesi Balestrin ${ }^{1,4}$, Lize Helena Cappellari² \& Arlete Ballestrin Outeiral ${ }^{3}$ \\ ${ }^{1}$ Laboratório de Herpetologia, Departamento de Zoologia, Instituto de Biociências, \\ Universidade Federal do Rio Grande do Sul - UFRS, \\ Av. Bento Gonçalves, 9500, prédio 43435, CEP 91501-970, Porto Alegre, RS, Brasil \\ ${ }^{2}$ Universidade da Região da Campanha - URCAMP, \\ Rua General Osório, 522, CEP 96570-000, Caçapava do Sul, RS, Brasil \\ ${ }^{3}$ Av. Arlindo Pasqualini, 480, Bairro Jardim Isabel, CEP 91760-140, Porto Alegre, RS, Brasil \\ ${ }^{4}$ Autor para correspondência: Rafael Lucchesi Balestrin,e-mail: atractus@hotmail.com
}

BALESTRIN, R.L., CAPPELLARI, L.H. \& OUTEIRAL, A.B. Reproductive biology of Cercosaura schreibersii (Squamata, Gymnophthalmidae) and Cnemidophorus lacertoides (Squamata, Teiidae) in Sul-Riograndense Shield, Brazil. Biota Neotrop. 10(1): http://www.biotaneotropica.org.br/v10n1/en/ abstract?article+bn02110012010.

\begin{abstract}
Gonads of 124 specimens of Cercosaura schreibersii (Wiegmann, 1834) and 192 specimens of Cnemidophorus lacertoides Duméril \& Bibron, 1839 from the Sul-Riograndense Shield, Rio Grande do Sul, Brazil were analyzed. Both species presented seasonal reproduction, with vitellogenic females found at the end of winter and spring, gravid females in spring and summer and recruitment recorded during the months with the higher mean temperatures in the region. Variation of testis volume was not observed in males of C. schreibersii throughout the year, while adult males of $C$. lacertoides had variation, with larger testis found in spring. All clutches of $C$. schreibersii comprised two eggs and data suggest positive correlation between female SVL and egg size $\left(\mathrm{R}^{2}=0.55 ; \mathrm{p}<0.05\right)$. Clutch size of $C$. lacertoides varied from 2 to 6 eggs $(3.75 \pm 1.24, \mathrm{n}=15)$ and positive correlation between female SVL and clutch size was also observed $\left(R^{2}=0.50 ; p<0.001\right)$. Females of C. schreibersii had significantly larger SVL than mature males ( $t$-test; $\mathrm{p}<0.0001)$. Head length and width of mature females and males of $C$. schreibersii were not significantly different. Sexually mature females of $C$. lacertoides had significantly larger SVL than mature males ( $t$ test; $p<0.001)$, however, males had larger head than females, with significant differences in head length (Ancova; $p<0.001$ ) and width (Ancova; $p<0.001$ ). Juvenile females can attain the size of mature specimens after seven months for C. schreibersii and eight months for C. lacertoides. It was not possible to estimate the sexual maturation age of males, but it is possible to estimate that males are mature earlier and with smaller size than females in both species.
\end{abstract}

Keywords: lizards, reproduction, Southern Brazil.

BALESTRIN, R.L., CAPPELLARI, L.H. \& OUTEIRAL, A.B. Biologia reprodutiva de Cercosaura schreibersii (Squamata, Gymnophthalmidae) e Cnemidophorus lacertoides (Squamata, Teiidae) no Escudo Sul-Riograndense, Brasil. Biota Neotrop. 10(1): http://www.biotaneotropica.org.br/v10n1/en/ abstract?article+bn02110012010.

Resumo: Foram analisadas as gônadas de 124 exemplares de Cercosaura schreibersii e 192 exemplares de Cnemidophorus lacertoides do Escudo Sul-Riograndense, Rio Grande do Sul, Brasil. Ambas as espécies apresentaram reprodução tipicamente sazonal com fêmeas vitelogênicas no final do inverno e primavera, ovígeras na primavera e verão e o recrutamento ocorrendo nos meses com médias mais altas de temperatura na região. Não foi observada variação no volume médio ajustado dos testículos de machos de $C$. schreibersii ao longo do ano, contudo, o volume médio ajustado dos testículos de machos adultos de $C$. lacertoides alcançaram os maiores valores na primavera. Todas as desovas de $C$. schreibersii apresentaram número fixo de 2 ovos $(\mathrm{n}=8)$ havendo correlação positiva entre o CRC das fêmeas e o tamanho dos ovos $\left(\mathrm{R}^{2}=0,55 ; \mathrm{p}<0,05\right)$. Em C. lacertoides o tamanho das desovas variou de 2 a 6 ovos $(\bar{x}=3,75 \pm 1,24 ; \mathrm{n}=15)$ e foi observada correlação positiva entre o CRC das fêmeas e o tamanho das desovas $\left(\mathrm{R}^{2}=0,50 ; \mathrm{p}<0,001\right)$. Em ambas as espécies, fêmeas maduras apresentam CRC significativamente maiores que machos (Teste $t ; \mathrm{p}<0,001$ ). Por outro lado, machos maduros apresentam o comprimento (Ancova; $p<0,001$ ) e largura da cabeça (Ancova; $p<0,001$ ) maiores que as das fêmeas. Fêmeas juvenis podem alcançar tamanho compatível ao de exemplares maduros em aproximadamente 7 meses de vida para $C$. schreibersii e 8 meses para $C$. lacertoides durante a primeira estação reprodutiva após o nascimento. Não foi possível estimar a idade de maturação sexual dos machos, contudo, pode-se inferir que amadureçam antes e com menor tamanho que as fêmeas em ambas as espécies.

Palavras-Chave: lagartos, reprodução, sul do Brasil. 


\section{Introdução}

Estudos descritivos sobre a reprodução de répteis têm fornecido dados essenciais para formular e testar diversas hipóteses de história de vida. Contudo, lagartos de áreas sob influência do clima temperado na região Neotropical têm sido pouco estudados, provavelmente em função da menor diversidade e, em alguns casos, pequena densidade que lhes é característica (Di-Bernardo et al. 2007).

Dentre os estudos realizados com espécies de lagartos em áreas de clima temperado na região Neotropical, destacam-se os com os teídeos Teius oculatus (Martori \& Acosta 1990, 1994, Bujes 1998a, b, 1999, Blanco \& Acosta 1998, Cappellari et al. 2007), Teius teyou (Cruz et al. 1999), Cnemidophorus lacertoides (Aún \& Martori 1996, Feltrim 2002), Cnemidophorus ocellifer (Cruz 1996) e o tropidurídeo Liolaemus occipitalis (Verrastro \& Bujes 1998, Verrastro \& Krause 1994, 1999).

Em recente revisão de Cercosaurinae, Doan (2003) sinonimizou os gêneros Pantodactylus e Prinodactylus a Cercosaura, redefinindoos para incluir 11 espécies e sete subespécies. A partir destas modificações, Cercosaura passou a ocorrer em quase todos os países da América do Sul (ausente somente no Chile) e na América Central. No sul do Brasil o gênero Cercosaura é representado pelas espécies C. schreibersi e C. ocellata. C. schreibersii (lagartixa-comum) distribui-se no sudeste do Peru, Bolívia, Paraguai, Argentina, Brasil meridional e Uruguai (Lema 1994). É a espécie mais comum de lagarto do Rio Grande do Sul, vivendo sob pequenas lascas de rochas em gramados (Cei 1986, Lema 1994, 2002). Informações acerca da biologia reprodutiva de Cercosaura são escassas e pontuais, representadas por citações fundamentadas em observações isoladas (Dixon \& Soini 1975, 1986, Gallardo 1977, Vitt 1991, Ávila-Pires 1995). Diehl (2007) estudou uma população de C. schreibersii no Planalto das Araucárias, no Estado do Rio Grande do Sul, e apresentou dados referentes ao ciclo reprodutivo, tamanhos que machos e fêmeas atingem a maturidade sexual, dimorfismo sexual no número de poros cloacais e em diversas medidas do corpo.

Cnemidophorus lacertoides é um lagarto teídeo de pequeno porte que ocorre na região sul do Brasil, Uruguai e nordeste da Argentina (Lema 1994, Peters \& Donoso-Barros 1970). No Rio Grande do Sul é encontrado no litoral norte, campos adjacentes e no Escudo SulRiograndense, geralmente associados a áreas rochosas (Lema 2002). Sobre a história de vida desta espécie, destacam-se os trabalhos de Aún \& Martori (1996), que descreveram a dieta e o ciclo reprodutivo de uma população na Argentina e Feltrim (2002) que analisou o dimorfismo sexual na morfologia externa da espécie.

Neste trabalho apresentamos dados referentes ao período reprodutivo, idade e tamanho de maturidade sexual e dimorfismo sexual no tamanho do corpo de Cercosaura schreibersii e Cnemidophorus lacertoides no Escudo Sul-Riograndense, Rio Grande do Sul, Brasil.

\section{Material e Métodos}

Os exemplares de Cercosaura schreibersii foram coletados em duas localidades, ambas localizadas no Escudo Sul-Riograndense. Entre janeiro de 2002 e dezembro de 2003 os animais foram capturados no Município de Dom Feliciano (Fazenda Chapada; $30^{\circ} 25^{\prime} 23,5^{\prime \prime} \mathrm{S}$ e $052^{\circ} 18^{\prime} 41,4$ " W altitude); enquanto que de abril de 2004 a abril de 2006, efetuaram-se amostras entre os Municípios de São Jerônimo e Barão do Triunfo (Fazenda Novosares; 30 22' 15,8" S e 51 54'07,4" O altitude). Os exemplares de C. lacertoides foram coletados somente na segunda localidade, nos meses de abril e dezembro do ano de 2003 e entre os meses de abril de 2004 a abril de 2006.

O Escudo Sul-Riograndense corresponde a um mosaico representado por zonas de transição de Campos e Floresta Estacional Semidecidual. A formação campestre apresenta fisionomia semelhan- te à Savana, com muitas pedras que, invadida por arbustos, originam os chamados campos "grossos". Em áreas úmidas, principalmente encostas e vales, há fragmentos de Floresta Estacional Semidecidual, caracterizada pela ausência de Apuleia leiocarpa (grápia) e presença de Araucaria angustifolia, Ocotea pulchella, Podocarpus lambertii e Ilex paraguariensis (Porto 2002, Quadros \& Pillar 2002).

Durante os anos de estudo, a precipitação média mensal no Escudo Sul-Riograndense variou de $29,6 \mathrm{~mm}^{3}$ a $320,6 \mathrm{~mm}^{3}$ ( $\bar{x}=147$, $81 \pm 35,28)$, enquanto que as temperaturas, de forma geral, foram elevadas no verão $\left(\bar{x}=21,85^{\circ} \mathrm{C} \pm 0,97\right)$ e comparativamente mais baixas no inverno ( $\bar{x}=14{ }^{\circ} \mathrm{C} \pm 1,27$ ) (Figura 1) (Fonte: INMET - Oitavo Distrito de Meteorologia - Porto Alegre, para Encruzilhada do Sul, município vizinho a área de estudo). O clima é de domínio exclusivo e quase absoluto Mesotérmico do tipo Temperado (IBGE 1992).

Os lagartos foram coletados manualmente, mortos no momento da captura, através de injeção letal do anestésico Tiopental ${ }^{\circledR}$, fixados com formol a $10 \%$ e posteriormente conservados em álcool $70 \%$, sendo depositados na coleção do Museu de Ciências e Tecnologia da Pontifícia Universidade Católica do Rio Grande do Sul (MCT) (Anexo 1). Todos os espécimes foram pesados com dinamômetro Pesola ${ }^{\circledR}$ (escala de $0,1 \mathrm{~g}$ ) e seu comprimento rostro-cloacal (CRC) e comprimento da cauda (CC) medidos com paquímetro digital Mitutoyo $^{\circledR}$ (escala de $0,1 \mathrm{~mm}$ ).

Para estimar o tamanho com que machos e fêmeas atingem a maturidade sexual foram considerados maduros machos portadores de canais deferentes e ou epidídimos enovelados, e fêmeas com comprimento rostro-cloacal igual ou maior que a menor fêmea portadora de folículos em vitelogênese secundária, com comprimento igual ou superior a $3 \mathrm{~mm}$, ou com ovos nos ovidutos.

Para estimar o período reprodutivo, machos e fêmeas tiveram suas gônadas analisadas e os dados referentes ao estado de maturação sexual das mesmas agrupados por mês de captura. Nos machos, o volume médio dos testículos foi calculado através da fórmula do volume do esferóide: Volume: 4/3(1/2 comprimento) (1/2 largura) ${ }^{2}$. Para que o CRC não influenciasse no volume dos testículos, foram calculados os resíduos das regressão entre o CRC e o volume dos testículos, realizando uma análise da distribuição mensal dos indivíduos adultos o volume ajustado do testículo ao longo dos anos do

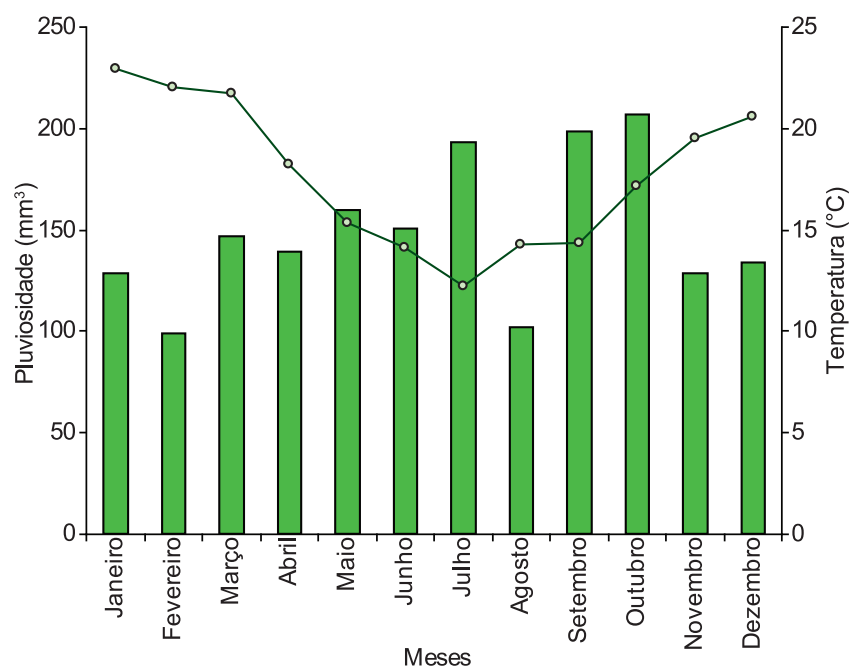

Figura 1. Temperatura e pluviosidade média mensal no Município de Encruzilhada do Sul (Escudo Sul-Riograndense), Rio Grande do Sul, de agosto de 2001 a abril de 2006. Barras = pluviosidade e linha = temperatura média.

Figure 1. Monthly mean temperature and rainfall in Encruzilhada do Sul municipality (Escudo Sul-Riograndense), Rio Grande do Sul, from August 2001 to April 2006. Bars = rainfall and line = monthly mean . 
estudo. Em fêmeas foi medido o comprimento do maior folículo ou ovo e este correlacionado aos diferentes meses de captura.

A idade com que os indivíduos atingem a maturidade sexual foi inferida a partir da correlação conjunta entre as épocas em que ocorrem nascimentos, o tamanho dos recém-nascidos (quando disponível), a distribuição das classes de tamanho ao longo do ano e o tamanho de maturação, para cada sexo. O tamanho dos recém-nascidos foi determinado através de espécimes nascidos em cativeiro e dados bibliográficos. Na tentativa de obter desovas e filhotes, alguns exemplares de C. schreibersii foram mantidos em cativeiro, possibilitando observações oportunísticas sobre o comportamento reprodutivo.

O tamanho das desovas foi estimado com base no número dos folículos vitelogênicos e/ou ovos presentes nas fêmeas. Fêmeas que apresentaram simultaneamente folículos vitelogênicos e ovos nos ovidutos foram consideradas como evidências de múltiplas desovas. Para analisar o tamanho e número de ovos em relação ao CRC das fêmeas, foi utilizado um teste de regressão simples. Para avaliar a existência de dimorfismo sexual foram utilizados apenas espécimes sexualmente maduros, independentemente das variáveis analisadas. Empregamos o $t$-teste (bi-caudal) para avaliar a existência de dimorfismo sexual entre o CRC de machos e fêmeas em ambas as espécies. Foi eliminado o efeito do CRC sobre as variáveis, comprimento e largura da cabeça de machos e fêmeas através de uma análise de regressão linear e uso dos resíduos. As regressões foram comparadas através da Análise da Covariância (Ancova, SPSS 10.0) para verificar o dimorfismo sexual no comprimento e largura da cabeça em ambas as espécies.

\section{Resultados}

\section{Tamanho do corpo e dimorfismo sexual}

Foram coletados 124 espécimes de Cercosaura schreibersii (72 machos e 52 fêmeas) e 192 exemplares de Cnemidophorus lacertoides (104 fêmeas, 83 machos e 5 espécimes sem confirmação do sexo).

O tamanho do CRC de fêmeas maduras de Cercosaura scherbersii variou de 34 , a $43,5 \mathrm{~mm}(\bar{x}=38 \pm 3 ; \mathrm{N}=32)$. Nos machos sexualmente maduros o CRC variou de 24 a $40,7 \mathrm{~mm}(\bar{x}=33,1 \pm 3,6$; $\mathrm{N}=68)$. Machos imaturos $(\mathrm{N}=4)$ foram capturados apenas nos meses de fevereiro e abril. Fêmeas maduras apresentaram CRC significativamente maior que machos maduros ( $t$-teste; $\mathrm{p}<0,0001)$. Foi observada diferença significativa entre o comprimento da cabeça (Ancova; $\mathrm{p}<0,01$ ) e largura da cabeça (Ancova; $\mathrm{p}<0,009$ ) de machos e fêmeas adultas de $C$. schreibersii.

Fêmeas sexualmente maduras de Cnemidophorus lacertoides mediram entre $54,4 \mathrm{~mm}$ a $76,9 \mathrm{~mm}$ de $\mathrm{CRC}(\bar{x}=67,3 \pm 5,6 ; \mathrm{N}=62)$. Machos maduros mediram entre $42,8 \mathrm{~mm}$ e $68,6 \mathrm{~mm}$ de CRC ( $\bar{x}=55,7 \pm 7 ; \mathrm{N}=69$ ). O CRC de fêmeas sexualmente maduras foi significativamente maior que o CRC de machos maduros ( $t$-teste; $\mathrm{p}<0,001)$. O comprimento da cabeça de machos sexualmente maduros foi significativamente maior que o das fêmeas maduras (Ancova; $\mathrm{p}<0,001$ ), assim como a largura da cabeça (Ancova; $\mathrm{p}<0,001$ ).

\section{Ciclo reprodutivo}

No Escudo Sul-Riograndense, fêmeas de Cercosaura schreibersii $(\mathrm{N}=52)$ apresentaram reprodução sazonal, sendo as vitelogênicas $(\mathrm{N}=16)$ observadas de agosto a novembro e as ovígeras $(\mathrm{N}=8)$ de outubro a dezembro e fevereiro (Figura 2). Todas as fêmeas apresentaram dois ovos nos ovidutos. Apesar do baixo número de fêmeas ovígeras na amostra, os dados sugerem correlação positiva entre o tamanho dos ovos e o CRC das fêmeas $\left(R^{2}=0,55 ; \mathrm{p}<0,05\right)$

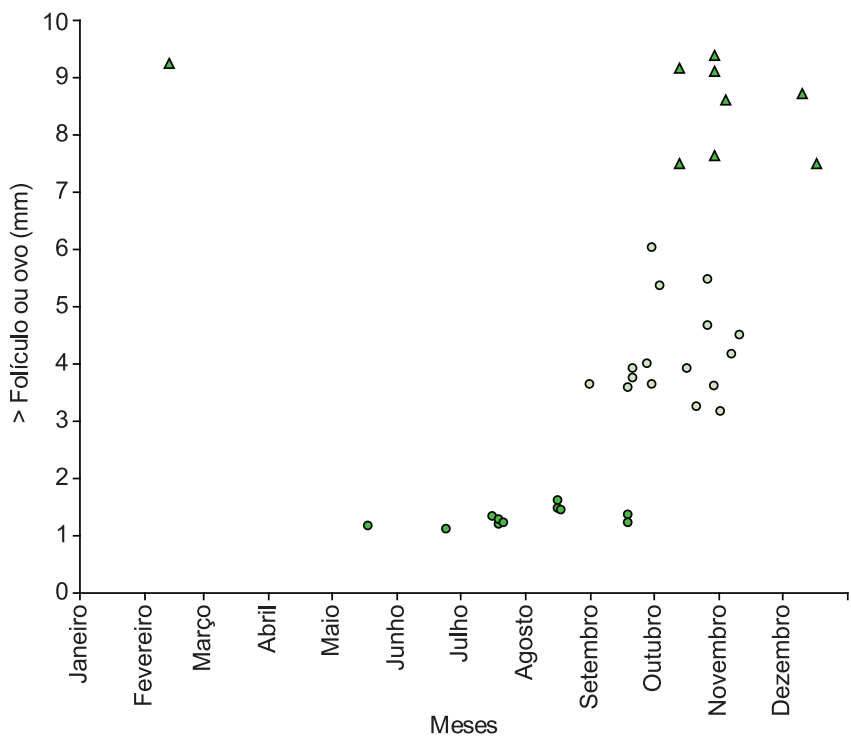

Figura 2. Variação sazonal no comprimento do maior folículo (círculos fechados $=$ folículos primários e círculos abertos $=$ folículos vitelogênicos) ou ovo (triângulos) de fêmeas maduras de Cercosaura schreibersii no sul do Brasil.

Figure 2. Seasonal variation of the larger ovarian follicles (closed circles $=$ non-vitellogenic follicles and open circles $=$ vitellogenic follicles) or eggs (triangles) of adult females of Cercosaura schreibersii in Southern Brazil.

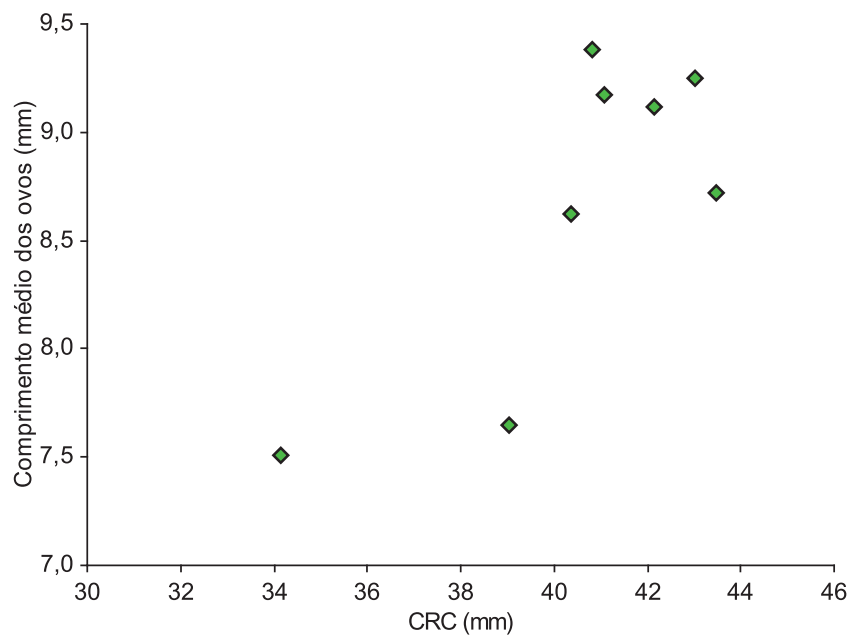

Figura 3. Relação entre o tamanho do ovo e o CRC de fêmeas de Cercosaura schreibersii no sul do Brasil.

Figure 3. Relationship between egg size and female body size (SVL) of Cercosaura schreibersii in Southern Brazil.

(Figura 3). Em uma das fêmeas ovígeras foram observados folículos vitelogênicos conjuntamente com ovos no oviduto, sugerindo múltiplas desovas. $\mathrm{O}$ menor espécime $(\mathrm{CRC}=17,8 \mathrm{~mm})$, provavelmente um recém-nascido, foi coletado no mês de dezembro. Outro juvenil com tamanho compatível ao de um filhote $(\mathrm{CRC}=20,7 \mathrm{~mm})$ foi coletado em fevereiro. A presença de juvenis em dezembro e fevereiro, assim como de fêmeas ovígeras nos meses de outubro, dezembro e fevereiro nos permite inferir que o recrutamento ocorra de dezembro a fevereiro, podendo se estender até o mês de março. Não foi observada variação no volume médio ajustado dos testículos de machos ao longo do ano.

Do total de fêmeas de Cnemidophorus lacertoides analisadas ( $\mathrm{N}=104)$, exemplares vitelogênicos $(\mathrm{N}=11)$ foram encontrados 
de outubro a dezembro e fêmeas ovígeras $(\mathrm{N}=5)$ de novembro a dezembro (Figura 4). O tamanho das desovas, estimado com base nos folículos vitelogênicos e ovos nos ovidutos, variou de 2 a 6 ovos $(\bar{x}=3,7 \pm 1,2 ; \mathrm{N}=15)$. Foi observada correlação positiva entre o CRC das fêmeas e o tamanho das desovas $\left(\mathrm{R}^{2}=0,50 ; \mathrm{p}<0,001\right)$ com fêmeas maiores tendendo a produzir um maior número de ovos (Figura 5). No mês de dezembro uma ninhada com quatro ovos foi encontrada na natureza sob pedra em uma colônia de fungos de um formigueiro de Acromyrmex Mayr, 1865. Junto, estava uma desova da serpente Taeniophallus occipitalis (Jan, 1863). Após 84 dias, no mês de março, nasceram três filhotes com CRC médio de 27,9 mm e massa média de $0,6 \mathrm{~g}$. Além dos filhotes nascidos em cativeiro, dois

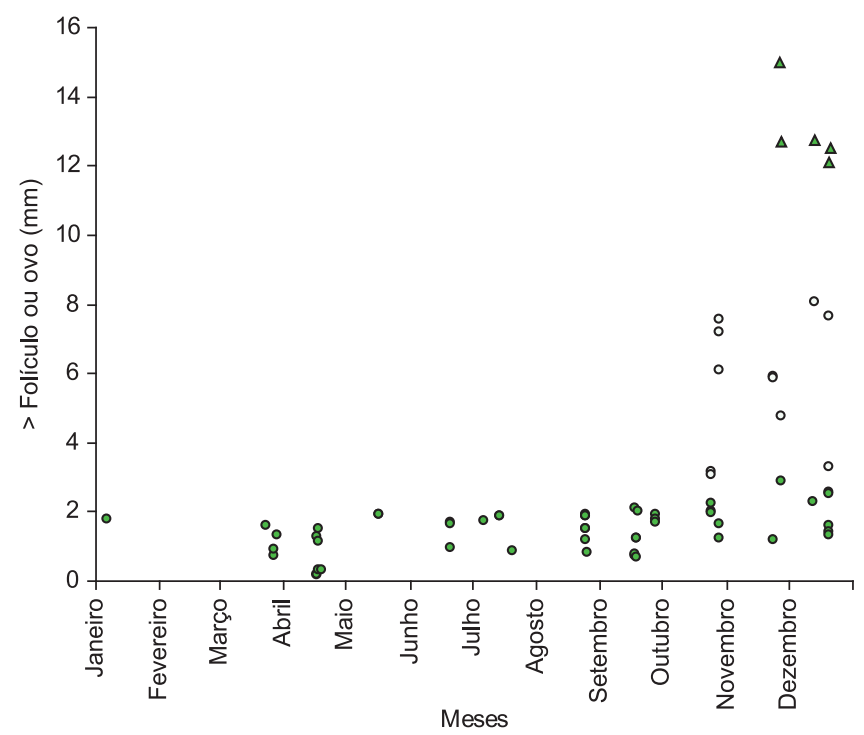

Figura 4. Variação sazonal no comprimentodo maior folículo (círculos fechados $=$ folículos primários; círculos abertos $=$ folículos vitelogênicos $)$ ou ovo (triângulos) de fêmeas maduras de Cnemidophorus lacertoides no sul do Brasil.

Figure 4. Seasonal variation of the larger ovarian follicles (closed circles $=$ non-vitellogenic follicles and open circles $=$ vitellogenic follicles) or eggs (triangles) of adult females of Cnemidophorus lacertoides in Southern Brazil.

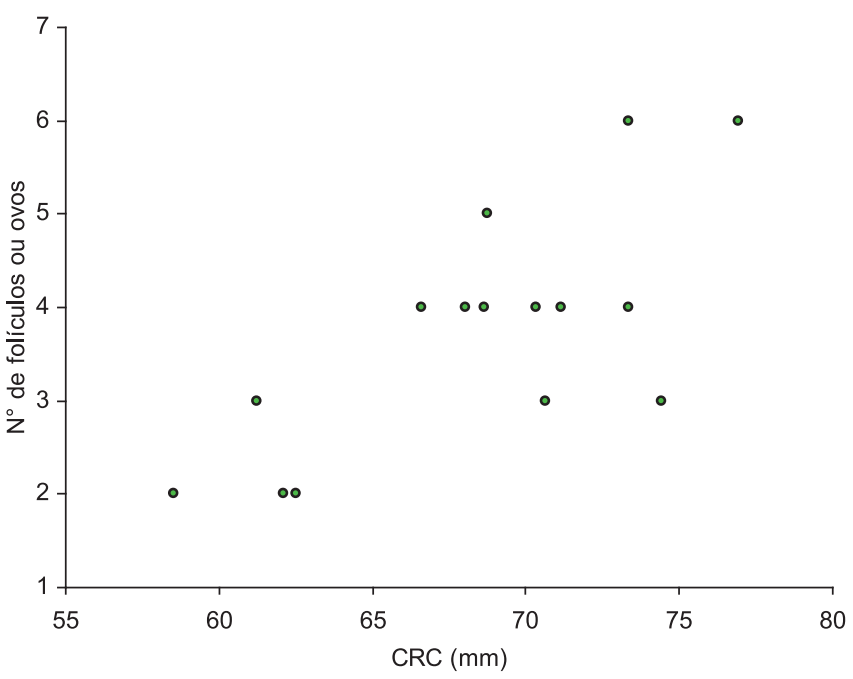

Figura 5. Relação entre o número de ovos e o CRC de fêmeas de Cnemidophorus lacertoides no sul do Brasil.

Figure 5. Relationship between clutch size and female body size (SVL) of Cnemidophorus lacertoides in Southern Brazil. espécimes com CRC próximo ao de recém nascidos foram observados em janeiro, sugerindo que o recrutamento ocorra nos meses de verão (janeiro, fevereiro e março). $\mathrm{O}$ volume médio ajustado dos testículos variou ao longo do ano com valores máximos em outubro e novembro, período em que foram encontradas fêmeas vitelogênicas e que precede o período em que foram encontradas fêmeas ovígeras, sugerindo que cópulas ocorram nestes dois meses. A partir do mês de dezembro o volume dos testículos diminuiu gradualmente até o mês de julho quando, voltou a aumentar (Figura 6).

\section{Maturidade sexual}

Uma análise conjunta entre o período estimado para o recrutamento de filhotes (dezembro a fevereiro), o tamanho estimado para a maturação sexual e a distribuição sazonal dos CRC de fêmeas de Cercosaura schreibersii permitiu inferir que algumas fêmeas podem atingir tamanho de indivíduos sexualmente maduros em aproximadamente sete meses, na primeira estação reprodutiva após seu nascimento (Figura 7), e com maior CRC que machos. No entanto, juvenis fêmeas de diferentes classes de CRC foram encontradas em um mesmo mês ao longo do ano. Em fevereiro foram encontrados juvenis com 29,1 e 31,8 mm de CRC, em abril com 25,9 e 32,7 mm de CRC, e em julho com 28,7 e $34 \mathrm{~mm}$ de CRC (Figura 7). Para machos, a distribuição sazonal dos CRC de C. schreibersii não permitiu inferir a idade com que estes atingem tamanho compatível ao de espécimes sexualmente maduros. Contudo, o baixo número de machos imaturos $(\mathrm{N}=4)$, em comparação com o total de indivíduos maduros $(\mathrm{N}=68)$, e o reduzido tamanho do menor macho maduro (24 mm) em relação às fêmeas maduras, indica que machos alcançam CRC de indivíduos sexualmente maduros precocemente, antes que as fêmeas.

Em uma análise conjunta do período de recrutamento, tamanho de maturação sexual e distribuição sazonal dos CRC podemos inferir que fêmeas de Cnemidophorus lacertoides podem alcançar o tamanho correspondente ao de espécimes sexualmente maduros em aproximadamente oito meses de vida, na primeira estação reprodutiva após seu nascimento (Figura 8). Contudo, da mesma forma que observado para Cercosaura schreibersii, juvenis fêmeas com diferentes tamanhos de CRC podem ser observados em um mesmo mês. Em

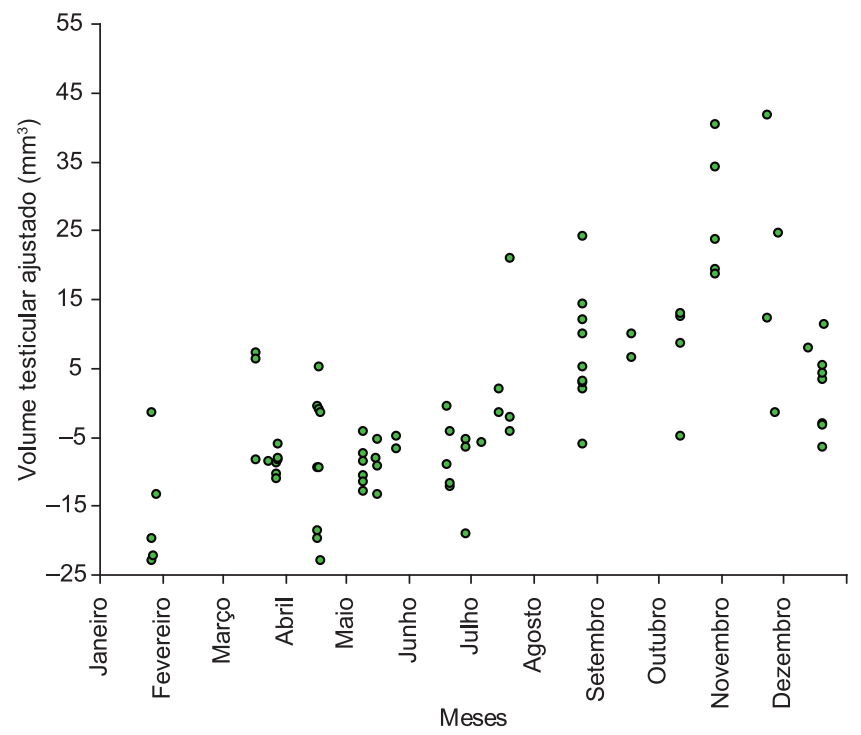

Figura 6. Variação sazonal do volume testicular residual $\left(\mathrm{mm}^{3}\right)$ de machos adultos de Cnemidophorus lacertoides no sul do Brasil.

Figure 6. Seasonal variation of a residual testes volume $\left(\mathrm{mm}^{3}\right)$ of adult males of Cnemidophorus lacertoides in Southern Brazil. 


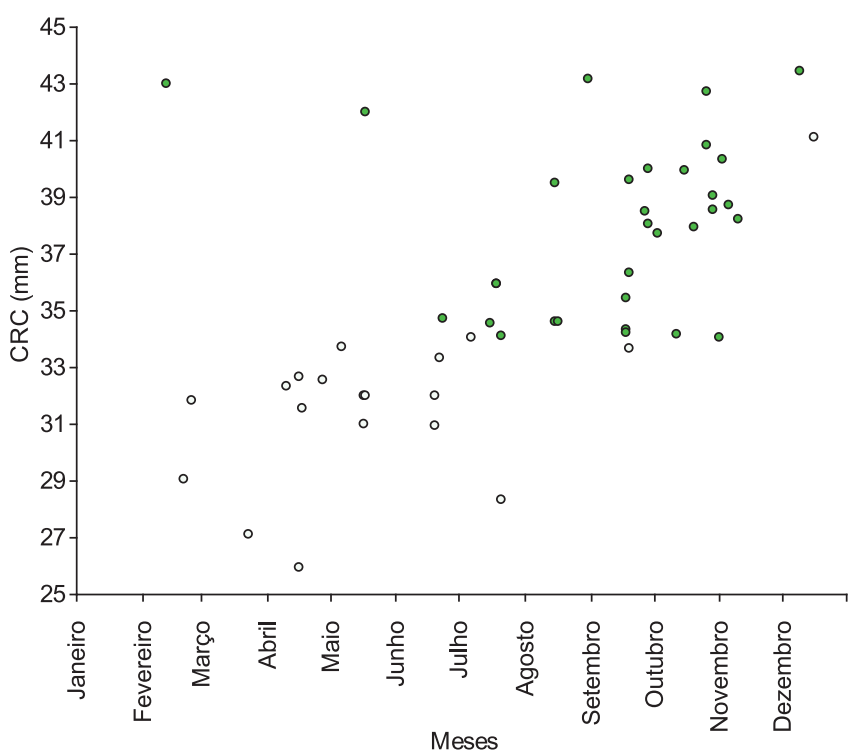

Figura 7. Distribuição sazonal do comprimento rostro-cloacal (CRC) em fêmeas de Cercosaura schreibersii no sul do Brasil. Círculos abertos = fêmeas imaturas; círculos fechados $=$ fêmeas maduras.

Figure 7. Seasonal distribution of snout-vent length (SVL) in females of Cercosaura schreibersii in Southern Brazil. Open circles = immature females; closed circles $=$ adult females.

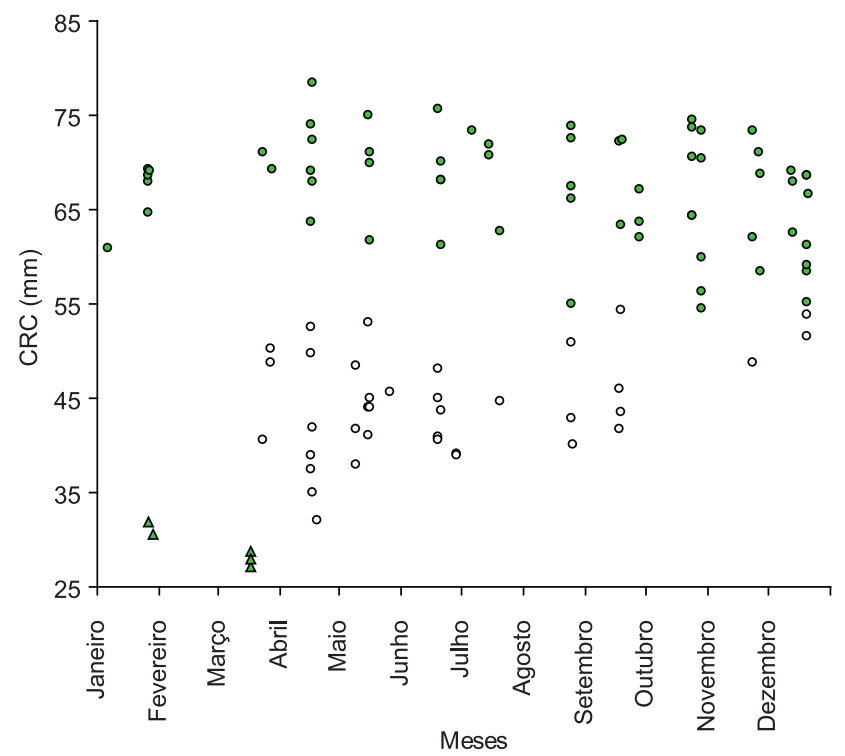

Figura 8. Distribuição sazonal do comprimento rostro-cloacal (CRC) em fêmeas de Cnemidophorus lacertoides no sul do Brasil. Triângulos = juvenis; círculos abertos = fêmeas imaturas; círculos fechados = fêmeas maduras.

Figure 8. Seasonal distribution of snout-vent length (SVL) in females of Cnemidophorus lacertoides in Southern Brazil. Triangles = juveniles; Open circles $=$ immature females; closed circles $=$ adult females.

março, podem-se observar desde filhotes recém eclodidos $(\mathrm{N}=3)$, com CRC médio de 27,9 mm até um juvenil com 50,2 mm de CRC, em abril identificaram-se juvenis com 32,1 até $52,5 \mathrm{~mm}$ de CRC, e em maio com 37,9 até $53 \mathrm{~mm}$ de CRC (Figura 8). Machos atingem a maturidade sexual com menor tamanho que fêmeas. Entretanto, também não foi possível estimar o tempo que juvenis machos levam para alcançar a maturidade sexual.

\section{Discussão}

\section{Tamanho do corpo e dimorfismo sexual}

O comprimento médio com que fêmeas $(\bar{x}=38 \pm 3 ; \mathrm{N}=32) \mathrm{e}$ machos maduros $(\bar{x}=33,1 \pm 3,6 ; \mathrm{N}=68)$ de Cercosaura schreiberssi atingem a maturidade sexual no Escudo Sul-Riograndense foi muito semelhante ao obtido por Diehl (2007) para fêmeas $(\bar{x}=40 \pm 0,2$, $\mathrm{N}=23)$ e machos ( $\bar{x}=32,6 \pm 0,31, \mathrm{~N}=34)$ de uma população desta espécie no Planalto das Araucárias. O bioma Mata Atlântica no Sul do Brasil apresenta formações campestres denominadas Campos de Altitude do Planalto das Araucárias ou Campos de Cima da Serra. Estas áreas predominam em zonas de maior altitude, com cotas superiores a $800 \mathrm{~m}$ e, diferentemente do Escudo Sul-Riograndense, são entremeados pela Floresta Atlântica com Araucária (Boldrini 2009).

Da mesma forma, fêmeas apresentaram CRC significativamente maior que o de machos adultos, em ambas as localidades. O tamanho do corpo é um fator importante que pode influenciar direta ou indiretamente vários aspectos reprodutivos das espécies de lagartos (Dunham et al. 1988, Vitt 1992). Por exemplo, fêmeas com maior tamanho do corpo podem ser favorecidas com a produção de maior volume e/ou tamanho da ninhada (Fitch 1970, Shine et al. 1998). No caso da espécie Cercosaura schreibersii, as desovas são compostas por um número fixo e reduzido de ovos, o que parece ser uma sinapomorfia da família Gymnophtalmidae (Fitch 1970, Vitt 1982, Ávila-Pires 1995). Desta forma, a maximização do esforço reprodutivo poderia ocorrer de duas maneiras não excludentes: investimento no tamanho dos ovos, ou investimento no número de ninhadas produzidas, o que parece especialmente verdadeiro para espécies de lagartos com número fixo de ovos por desova (Andrews \& Rand 1974, Vitt 1986, Selcer 1990, Doughty 1997). Apesar do baixo número de fêmeas prenhes na amostra $(\mathrm{n}=8)$, os dados sugerem uma correlação positiva entre o CRC de fêmeas de C. schreibersii e o tamanho de seus ovos, de maneira que espécimes de maior CRC conseguiriam produzir ovos maiores do que aquelas de menor CRC. Foi sugerida a possibilidade de ocorrerem múltiplas desovas (ver ciclo reprodutivo) e isto também foi observado para a espécie no Planalto das Araucárias (Diehl 2007). Assim, a relação positiva entre o CRC e o tamanho dos ovos, bem como múltiplas desovas em um ciclo reprodutivo sazonal poderiam explicar, pelo menos em parte, o maior tamanho das fêmeas em relação aos machos de C. schreibersii. Outra hipótese a ser considerada, na tentativa de explicar o maior tamanho das fêmeas de $C$. schreibersii em relação aos machos, admitiria uma relação de custo benefício entre fecundidade e locomoção. Alguns aspectos da reprodução de $C$. schreibersii são muito semelhantes ao observado para o pequeno "lagarto-voador" Draco melanopogon, onde fêmeas apresentam maior CRC que machos e um número fixo e reduzido de ovos (2 ovos) por desova (Shine et al. 1998). Para esta espécie foi sugerido que a evolução na locomoção (vôo) poderia ter aumentado o grau em que a gravidez afetaria a habilidade locomotora das fêmeas ovígeras. Desta forma, um número reduzido de ovos favoreceria o "vôo" na espécie. Apesar de C. schreibersii ser um lagarto estritamente terrestre, possui um corpo extremamente alongado, ofioforme, que favorece sua rápida locomoção sob denso gramado e por entre pequenas rochas de afloramentos graníticos do Escudo Sul- Riograndense. O formato ofioforme do corpo parece comum a todas as espécies da família Gymnophtalmidae (Doan 2003). Diehl (2007) observou interessantes e significativas diferenças no tamanho dos membros e distância entre os membros posteriores e anteriores de machos e fêmeas em uma população do Planalto das Araucárias, onde machos apresentaram os membros significativamente mais longos do que os das fêmeas de mesmo CRC e fêmeas apresentaram maior 
distância entre os membros anteriores e posteriores do que aquela observada para machos de mesmo CRC. Estes dados sugerem que fêmeas de C. schreibersii estariam mais adaptadas a deslizar que os machos por estarem mais próximas do solo e serem mais alongadas. Desta forma o maior tamanho do CRC das fêmeas de C. schreibersii, associado a um número fixo, reduzido e um par de ovos por desova poderia estar refletindo uma relação de custo benefício entre fecundidade e locomoção como sugerido para D. melanopogon, onde fêmeas maiores teriam maior aptidão em carregar suas desovas, compostas por poucos, porém grandes ovos, e fugir de eventuais predadores (Shine et al. 1998). Relações entre fecundidade e locomoção também foram sugeridas para outros répteis Squamata, como anfisbenídeos e serpentes fossórias, onde a baixa fecundidade e o formato alongado dos ovos poderiam estar favorecendo o deslocamento de fêmeas prenhes por galerias sob o solo (Colli \& Zamboni 1999, Andrade et al. 2004, Balestrin \& Di-Bernardo 2005). O baixo número de fêmeas ovígeras coletadas pode estar relacionado ao método de coleta, já que todos os exemplares foram coletados manualmente nas áreas de interesse. Fêmeas ovígeras podem ter sua capacidade de locomoção reduzida por ocasião da gravidez e, consequentemente, estarem menos disponíveis a captura por métodos de procura ativa. Muitos autores sugerem que esta redução na locomoção pode estar associada ao aumento da carga física por ocasião dos ovos ou filhotes. No entanto, Olsson et al. (2000), estudando o lagartos scincídio Niveoscincus microlepidotum, observaram que, para esta espécie, não ocorre um efeito direto da carga física na locomoção, por ocasião da gravidez. Neste caso, modificações fisiológicas no organismo ou, simplesmente, o desgaste causado pela gestação, poderiam sustentar de forma mais adequada e explicar a redução da capacidade locomotora por fêmeas prenhes.

Apesar de menores em CRC que fêmeas, machos maduros apresentam cabeças significativamente mais compridas e largas que as fêmeas. Diehl (2007) também observou estas diferenças para machos e fêmeas do Planalto das Araucárias. As maiores dimensões da cabeça de machos em relação à das fêmeas, poderiam ser explicadas por duas hipóteses não excludentes. A primeira cita que machos com cabeças maiores são mais eficientes nas interações agressivas e apresentam maior habilidade para segurar as fêmeas durante a cópula (Anderson \& Vitt 1990). No cativeiro pode-se observar este comportamento em três cópulas de Cercosaura schreibersii. Além disso, também foram observadas interações agonísticas entre machos na disputa por fêmeas. A outra hipótese a ser admitida seria que as maiores dimensões da cabeça de machos atuariam no sentido de minimizar a competição intersexual por recursos alimentares (Van Sluys 1993), o que não foi testado neste estudo.

Cnemidophorus lacertoides também apresentou dimorfismo sexual no CRC entre machos e fêmeas sexualmente maduros, onde fêmeas foram significativamente maiores que machos, diferente do observado para muitos teídeos (Anderson \& Vitt 1990) e do descrito por Feltrim (2002) para C. lacertoides, que não observou diferenças significativas para esta variável. Como já mencionado, fêmeas com maior tamanho do corpo podem ser favorecidas com a produção de maior volume e/ou tamanho da ninhada (Fitch 1970, Shine et al. 1998). Os dados revelaram correlação positiva entre o CRC de fêmeas e o tamanho das desovas. Assim, fêmeas de C. lacertoides com maior CRC conseguiriam produzir mais ovos do que fêmeas de menor tamanho. A ausência de dimorfismo sexual observada por Feltrim (2002) entre o CRC de machos e fêmeas de $C$. lacertoides poderia ser justificada pelo pequeno tamanho da amostra. A autora utilizou um número reduzido de espécimes $(\mathrm{N}=47)$, em comparação com este estudo ( $\mathrm{N}=125)$, os quais abrangeram uma ampla distribuição geográfica (Uruguai, Argentina e Brasil), bem como diferentes ambientes, refletindo populações distintas. Adicionalmente, Feltrim (2002) estimou a idade da maturação sexual em $40 \mathrm{~mm}$ de CRC para machos e fêmeas, diferentemente do observado nesse trabalho (42,8 $\mathrm{mm}$ para machos e 54,4 mm para fêmeas), o que pode indicar a inclusão de indivíduos imaturos em sua análise de dimorfismo sexual.

Os dados revelaram que machos maduros de Cnemidophorus lacertoides apresentaram comprimento e largura da cabeça significativamente maiores do que aqueles das fêmeas sexualmente maduras. Estas diferenças parecem comuns entre machos e fêmeas de lagartos teídeos (Anderson \& Vitt 1990), estando de acordo com o observado por Feltrim (2002) para C. lacertoides. Não foram observados encontros agonísticos e cópulas para $C$. lacertoides, mas isto já foi citado para várias espécies do gênero Cnemidophorus (e.g. C. ocellifer; Vitt 1983), bem como para outros lagartos teídeos como Teius oculatus (Cappellari 2005) no sul do Brasil. A dieta de C. lacertoides não foi analisada, não sendo possível fazer qualquer inferência acerca desta variável em relação às diferenças observadas na largura e comprimento da cabeça entre machos e fêmeas.

\section{Ciclo reprodutivo}

No Escudo Sul-Riograndense a reprodução de Cercosaura schreibersii é sazonal, com o aparecimento de fêmeas vitelogênicas no final do mês de agosto até novembro e fêmeas ovígeras de outubro a fevereiro. Diehl (2007) também observou uma reprodução marcadamente sazonal para a espécie no Planalto das Araucárias. A maioria dos gimnoftalmídeos possui uma ampla estação reprodutiva (Vitt 1982), por exemplo, fêmeas de Cercosaura ocellata, no Peru, estão ovígeras durante seis meses do ano (setembro a fevereiro) (Dixon \& Soini 1986). Contudo, o clima Mesotérmico temperado da região sul do Brasil parece impor restrições fisiológicas que limitam a atividade de diversas espécies de répteis (Maciel et al. 2004, Hartmann et al. 2004, Aguiar \& Di-Bernardo 2005, Balestrin \& Di-Bernardo 2005, Cappellari 2005) aos meses quentes do ano. Assim, muito do que é sabido para répteis de regiões tropicais do Brasil pode não ser útil para as populações ou espécies proximamente relacionadas no sul do Brasil (Di-Bernardo et al. 2007). O recrutamento de juvenis de C. schreibersii ocorre nos meses com médias mais altas de temperatura na região, época em que as condições ambientais parecem ser mais favoráveis para o desenvolvimento dos filhotes (Romero-Schmidt \& Ortega-Rubio 2000). Em climas frios o sincronismo da eclosão com circunstâncias ambientais benignas podem contribuir com uma maior taxa de sobrevivência de filhotes, sendo isto especialmente verdadeiro para espécie com tamanho fixo de desovas, em que as fêmeas podem ajustar o tamanho do ovo e a frequiência da reprodução (Boretto \& Ibarguengoyt1a 2006). Isso parece especialmente verdadeiro para C. schreibersii, pois fêmeas apresentam número fixo de ovos por desova e múltiplas desovas podem ocorrer durante o ano. Múltiplas desovas podem maximizar um período reprodutivo curto (sazonal) como já foi sugerido para serpentes de regiões temperadas (Aldridge 1979). No caso de C. schreibersii, múltiplas desovas e filhotes proporcionalmente grandes em relação ao tamanho dos adultos poderiam compensar um ciclo reprodutivo curto (sazonal), desovas pequenas e auxiliar na rápida maturação sexual, garantindo o equilíbrio populacional da espécie. Neste estudo foram coletados dois espécimes de $C$. schreibersii com tamanho compatível com o de recém-nascidos (17,8 e 20,7 mm), o que é sustentado por Diehl (2007) que observou um tamanho médio de 18,3 \pm 0,1 mm para recém-nascidos em cativeiro do Planalto das Araucárias. Estes dados indicam que recém-nascidos eclodem relativamente grandes em comparação com CRC dos adultos, o que poderia contribuir com a rápida maturação sexual observada na espécie neste estudo. A redução na fecundidade está, geralmente, correlacionada com a seleção para que o maior tamanho do corpo ao nascer realce a sobrevivência do juvenil (Piantoni et al. 2006). 
Cnemidophorus lacertoides também apresentou um ciclo reprodutivo caracteristicamente sazonal, com uma estação reprodutiva que vai de outubro a dezembro e recrutamento de janeiro a fevereiro, coincidindo com os períodos de temperatura mais elevada na região e concordando com o observado por Aún \& Martori (1996) para a espécie na Argentina. Os lagartos teídeos da região do Chaco e de outras zonas temperadas apresentam atividade reprodutiva cíclica, e estes ciclos podem depender de chuva, da disponibilidade de alimento e dos dias com condições ambientais favoráveis (Martori \& Aún 1993, Martori \& Acosta 1994). Neste caso a temperatura parece ser um dos principais fatores determinantes da sazonalidade reprodutiva da espécie, assim como sugerido para C. schreibersii neste estudo e para outros lagartos de regiões temperadas neotropicais (Cruz 1996, Cruz et al. 1999). O tamanho da desova de C. lacertoides na área deste estudo também esta de acordo com o observado por Aún \& Martori (1996) para a espécie na Argentina. Da mesma forma que estes autores, não encontramos indícios de múltiplas desovas, sugerindo que $C$. lacertoides produza apenas uma desova por estação reprodutiva. $\mathrm{O}$ curto período de atividade reprodutiva de $C$. lacertoides (aproximadamente três meses entre o aparecimento de fêmeas vitelogênicas e ovígeras), em relação ao observado para C. schreibersii, provavelmente limita o tempo para investimentos em mais de uma desova por estação reprodutiva. Em teídeos de distribuição latitudinal mais ampla, podemos observar variações no período reprodutivo de acordo com o clima da região. A exemplo disso, Cnemidophorus ocellifer apresenta ciclo reprodutivo contínuo com múltiplas desovas em áreas de clima tropical e ciclo sazonal com recesso invernal em área de clima temperado (Vitt \& Breitenbach 1993, Aún \& Martori 1996). Com base nos dados observados, o fato de $C$. lacertoides investir em um maior número de ovos por desova pode contribuir com a manutenção da população local sem a necessidade de um maior investimento em desovas adicionais na mesma estação reprodutiva, diferente do observado para $C$. schreibersii que apresenta número reduzido e fixo de ovos por desova.

\section{Maturidade sexual}

A análise conjunta entre o período de recrutamento, tamanho de maturação sexual e distribuição sazonal dos CRC de fêmeas de Cercosaura schreibersii permite estimar que juvenis possam alcançar o tamanho de indivíduos maduros em aproximadamente sete meses após a eclosão dos ovos, na primeira estação reprodutiva após seu nascimento. Contudo, diferentes classes de CRC foram encontradas em um mesmo mês entre juvenis. Padrão semelhante de maturidade sexual foi observado para $C$. lacertoides, onde fêmeas podem atingir o tamanho de indivíduos sexualmente maduros em aproximadamente oito meses de idade, na primeira estação reprodutiva após seu nascimento, sendo que diferentes classes de tamanho de juvenis também foram observadas em um mesmo mês. As diferentes classes de CRC, observadas em juvenis capturados no mesmo mês, podem estar relacionada ao período de nascimento, o que poderia determinar diferenças na idade de maturação destes indivíduos (ver Andrews 1982). Por exemplo, filhotes de C. schreibersii provenientes das primeiras desovas (outubro, novembro) poderiam alcançar tamanho compatível ao de indivíduos maduros na primeira estação reprodutiva após seu nascimento. Filhotes provenientes de desovas tardias (janeiro e fevereiro) poderiam não alcançar o tamanho compatível com o de indivíduos sexualmente maduros na primeira estação reprodutiva, tornando-se maduros no segundo ano de vida. O ciclo reprodutivo de $C$. lacertoides é mais curto do que aquele observado para C. schreibersii, com desovas ocorrendo em apenas dois meses (novembro e dezembro). Contudo, diferenças entre a idade de maturação observadas nos juvenis também podem estar relacionadas ao período que estes nasceram, a exemplo do que foi observado para
Teius oculatus (Cappellari 2005). Padrão semelhante de maturação sexual também foi observado para duas espécies de serpentes, Lystrophis dorbignyi (Oliveira 2005) e Atractus reticulatus (Balestrin \& Di-Bernardo 2005) no sul do Brasil.

Para ambas as espécies não foi possível estimar a idade que machos alcançam tamanho compatível ao de indivíduos sexualmente maduros. Contudo, foi possível estimar que atinjam a maturidade sexual antes das fêmeas e com menor tamanho. O menor tamanho dos machos poderia estar relacionado a um menor esforço reprodutivo em comparação com o das fêmeas (Cox et al. 2003). Paralelamente, o fato de machos amadurecerem precocemente, antes que as fêmeas reduziria o risco de fêmeas copularem com machos inférteis, garantindo a manutenção dos estoques de machos disponíveis por período reprodutivo (Olsson \& Madsen 1996).

\section{Agradecimentos}

Agradecemos a Gilson Natal Galera e José Carlos Costa Karst por facilitar e permitir o trabalho na Fazenda Novosares, e a Custódio de Almeida na Fazenda Chapada. Somos gratos também a Alfredo Santos-Jr., Fabrício Bonfiglio e Síria B. Ribeiro pela revisão e auxílio na confecção deste manuscrito. Este trabalho foi financiado pelo Conselho Nacional de Desenvolvimento Científico e Tecnológico (CNPq) para RLB.

\section{Referências}

AGUIAR, L.F.S. \& DI-BERNARDO, M. 2005. Reproduction of the wather snake Helicops infrataeniatus (Colubridae) in southern, Brazil. AmphibiaReptilia 26(4):527-533.

ALDRIDGE, R.D. 1979. Female reproductive cycles in the snakes Arizona elegans and Crotalus viridis. Herpetologica 35:256-261.

ANDERSON, R.A. \& VITT, L.J. 1990. Sexual selection versus alternative causes of sexual dimorphism in the teiid lizards. Oecologia 84:145-157.

ANDRADE, D.V., SANDERS, C., MILSOM, W.K. \& ABE, A.S. 2004. Overwintering in Tegu Lizards. In Life in the cold: evolution, mechanisms, adaptation, and application. Twelfth International Hibernation Symposium (B.M. Barnes \& H.V. Carey, eds.). University of Alaska, Alaska. p. 339-340. (Biological Papers n. 27)

ANDREWS, R. \& RAND, S.A. 1974. Reproductive effort in anoline lizards. Ecology 55(6):1317-1327.

ANDREWS, R.M. 1982. Patterns of growth in reptiles. In Biology of the Reptilia (C. Gans \& F.H. Pough, eds.). Academic Press, New York. P .273-320.

AÚN, L. \& MARTORI, R. 1996. Características de la biología de Cnemidophorus serranus y Cnemidophorus lacertoides. Cuad. Herpetol. 9:95-99.

ÁVILA-PIRES, T.C.S. 1995. Lizards of Brazilian Amazonia (Reptilia: Squamata). Zool. Verhandel. 299:1-706.

BALESTRIN, R.L. \& DI-BERNARDO, M. 2005. Reproductive biology of Atractus reticulatus (Boulenger, 1885) (Serpentes - Colubridae) in southern Brazil. J. Herpetol. 15(3):195-199.

BLANCO, G.M. \& ACOSTA, J.C. 1998. Ecología reproductiva de Teius oculatus en la provincia de Córdoba, Argentina. Bol. Soc. Biol. Concepción 69:33-38.

BOLDRINI, I.I. 2009. Biodiversidade dos Campos do Planalto das Araucárias. MMA, Brasília, 240 p. (Série Biodiversidade v. 30)

BORETTO, J. \& IBARGUENGOYTIA, N. 2006. Asynchronous spermatogenesis and biennial female cycle of the viviparous lizard Phymaturus antofagastensis: reproductive responses to high altitudes and temperate climate of Catamarca, Argentina. Amphibia-Reptilia 12:25-36.

BUJES, C.S. 1998a. Mating behavior of Teius oculatus (Sauria, Teiidae). Amphibia-Reptilia 19:220-223.

BUJES, C.S. 1998b. Padrões de atividade de Teius oculatus (Sauria, Teiidae) na Reserva Biológica do Lami, Estado do Rio Grande do Sul - Brasil. Cuad. Herpetol. 12(2):13-21.

BUJES, C.S. 1999. Notes on the burrow construction by the Teius oculatus (Sauria, Teiidae). Biociências 7(1):51-56.

CAPPELLARI, L.H. 2005. História natural de Teius oculatus (Sauria: Teiidae) no sul do Brasil (Dom Feliciano, Rio Grande do Sul). Tese de Doutorado, 
Pontifícia Universidade Católica do Rio Grande do Sul, Porto Alegre, $58 \mathrm{p}$.

CAPPELLARI, L.H., LEMA, T., PRATES, P. \& ROCHA, C.F.D. 2007. Diet of Teius oculatus (Sauria, Teiidae) in southern Brazil (Dom Feliciano, Rio Grande do Sul). Iheringia, Ser. Zool. 97(1):31-35.

CEI, M.J. 1986. Reptiles del Centro, Centro-Oeste y Sur de la Argentina. HerpetofAúna de Zonas Áridas y Semiáridas. Museo Regionale di Scienze Naturali, Italia, 327 p. (Monografía IV).

COLLI, G.R. \& ZAMBONI, D.S. 1999. Ecology of the worm-lizard Amphisbaena alba in the Cerrado of central Brazil. Copeia 1999(3):733-742.

COX, R.M., SKELLY, S.L. \& JOHN-ALDER, H.B. 2003. A comparative test of adaptative hypotheses for sexual size dimorphism in lizards. Evolution 57(7):1653-1669.

CRUZ, F. 1996. Reproductive biology of the lizard Cnemidophorus ocellifer in the dry chaco of Salta, Argentina. Amphibia-Reptilia 17:80-86.

CRUZ, F.B., TEISAIRE, E., NIETO, L. \& ROLDÁN, A. 1999. Reproductive biology of Teius teyou in the semiarid chaco of Salta, Argentina. J. Herpetol. 33(3):420-429.

DI-BERNARDO, M., BORGES-MARTINS, M., OLIVEIRA, R.B. \& PONTES, G.M.F. 2007. Taxocenoses de serpentes de regiões temperadas do Brasil. In Herpetologia no Brasil 2 (L.B. Nascimento, A.T. Bernardes \& G.A. Cotta, eds.). PUCMG, Belo Horizonte, p. 222-263.

DIEHL, L.S. 2007. Biologia reprodutiva de Cercosaura schreibersii (Wiegmann, 1834) (Sauria: Gymnophthalmidae) no sul do Brasil. Dissertação de Mestrado, Pontifícia Universidade Católica do Rio Grande do Sul, Porto Alegre, $42 \mathrm{p}$.

DIXON, J.R. \& SOINI, P. 1975. The reptiles of the upper Amazon basin, Iquitos region, Peru. Part I. Lizards and Amphisbaenians. Milwaukee Publ. Mus. Contrib. Biol. Geol. 4:1-58.

DIXON, J.R. \& SOINI, P. 1986. The reptiles of the Upper Amazon Basin, Iquitos Region, Peru. I Lizards and amphisbaenians. II Crocodilians turtles and snakes. Milwaukee Public Museum, Milwaukee, $154 \mathrm{p}$.

DOAN, T.M. 2003. A new phylogenetic classification for the gymnophthalmid genera Cercosaura, Pantodactylus, and Prionodactylus (Reptilia: Squamata). Zool. J. Linn. Soc. 137:101-115.

DOUGHTY, P. 1997. The effects of "Fixed" clutch sizes on lizards life-histories: reproduction in the Australian velvet gecko, Oedura lesueurii. J. Herpetol. 31(2):266-272.

DUNHAM, A.E., MILES, D.B. \& REZNICK, D.N. 1988. Life history patterns in squamate reptiles. In Biology of the Reptilia (C. Gans \& R. B. Huey, eds.). Alan R. Liss, Inc., New York, p. 441-522. (v. 16)

FELTRIM, A.C. 2002. Dimorfismo sexual em Cnemidophorus lacertoides (Squamata, Teiidae) do Sul da América do Sul. Phyllomedusa 1(2):75-80.

FITCH, H.S. 1970. Reproductive cycles in lizards and snakes. University of Kansas, Kansas, p. 1-247. (Miscellaneous Publication n. 52).

GALLARDO, J.M. 1977. Reptiles de los alrededores de Buenos Aires. EUDEBA, Buenos Aires, $213 \mathrm{p}$

HARTMANN, T.M., MARQUES, O.A.V. \& ALMEIDA-SANTOS, S.M. 2004 Reproductive biology of the southern Brazilian pitviper Bothrops neuwiedi pubescens (Serpentes, Viperidae). Amphibia-Reptilia 25(1):77-85.

INSTITUTO BRASILEIRO DE GEOGRAFIA E ESTATÍSTICA - IBGE. 1992. Manual Técnico da Vegetação Brasileira. Rio de Janeiro. 92 p. (Séries Manuais Técnicos em Geociências n. 1)

LEMA, T. 1994. Lista comentada dos répteis ocorrentes no Rio Grande do Sul, Brasil. Comun. Mus. Ciênc. Tecnol. PUCRS Sér. Zool. 7:41-150.

LEMA, T. 2002. Répteis recentes do Rio Grande do Sul. In Os répteis do Rio Grande do Sul: atuais e fósseis - biogeografia - ofidismo. (T. Lema, ed.). EDIPUCRS, Porto Alegre, p. 35-91.

MACIEL, A.P., DI-BERNARDO, M., HARTZ, S.H., OLIVEIRA, R.B. \& PONTES, G.M.F. 2004. Seasonal and daily activity patterns of Liophis poecilogyrus (Serpentes: Colubridae) on the north coast of Rio Grande do Sul, Brazil. Amphibia-Reptilia 24(2):189-200.

MARTORI, R. \& ACOSTA, J.C. 1990. Ecología de una populación de Teius oculatus de Rio Cuarto (Córdoba) I - Estructura poblacional y crecimiento individual. Cuad. Herpetol. 5(3):15-18.

MARTORI, R. \& ACOSTA, J.C. 1994. Variación anual de la estructura de una población de Teius oculatus (Sauria, Teiidae) en Río Cuarto, Argentina. Rev. Esp. Herpetol. 8:37-44.

MARTORI, R. \& AÚN, L. 1993. Reproductive cycle of the parthenogenetic lizard Teius suquiensis. Amphibia-Reptilia 14(4):389-393.
OLIVEIRA, R.B. 2005. História natural da comunidade de serpentes de uma região de dunas do Litoral Norte do Rio Grande do Sul, Brasil. Tese do Doutorado, Pontifícia Universidade Católica do Rio Grande do Sul, Porto Alegre, $108 \mathrm{p}$.

OLSSON, M. \& MADSEN, T. 1996. Costs of mating with infertile males selects for late emergence in female sand lizards (Lacerta agilis L.). Copeia 2:462-464.

OLSSON, M., SHINE, R. \& BAK-OLSSON, E. 2000. Locomotor impairment of gravid lizards: is the burden physical or physiological? J. Evol. Biol. 13(2):263-268.

PETERS, J.A. \& DONOSO-BARROS, R. 1970. Catalogue of the Neotropical Squamata. II. Lizards and amphisbaenians. US Nat. Mus. Bull. 297:1293.

PIANTONI, C., IARGUENGOYTIA, N. \& CUSSAC, V. 2006. Growth and age of the southernmost nocturnal Gecko Homonota darwini: a skeletochronological assessment. Amphibia-Reptilia 27:393-400.

PORTO, M.L. 2002. Os campos sulinos, sustentabilidade e manejo. Cienc. Ambient. 1:119-138.

QUADROS, F.L.F. \& PILLAR, V.P. 2002. Transições floresta-campo no Rio Grande do Sul. Cienc. Ambient. 1:109-118.

ROMERO-SCHMIDT, H.L. \& ORTEGA-RUBIO, A. 2000. Reproduction of the Cape Orange-throat Whiptail, Cnemidophorus hyperythrus hyperythrus. Herpetol. Nat. Hist. 7(1):1-7.

SELCER, K.W. 1990. Egg-size relationship in a lizard with fixed clutch size: variation in population of the Mediterranean gecko. Herpetologica 46:15-21.

SHINE, R., KEOGH, S., DOUGHTY, P. \& GIRAGOSSYAN, H. 1998. Costs of reproduction and the evolution of sexual dimorphism in a "flaying lizard" Draco melanopogon (Agamidae). J. Zool. 246:203:213.

Van SLUYS, M. 1993. The reproductive cycle of Tropidurus itambere (Sauria: Tropiduridade) in Southeastern Brazil. J. Herpetol. 27(1):28-32.

VERRASTRO, L. \& BUJES, C.S. 1998. Ritmo de atividade de Liolaemus occipitalis Boulenger (Sauria, Tropiduridae) na Praia de Quintão, RS, Brasil. Rev. Bras. Zool. 15(4):913-920.

VERRASTRO, L. \& KRAUSE, L. 1994. Analysis of growth in a population of Liolaemus occipitalis Boul.1885, from the coastal sand-dunes of Tramandaí, RS, Brazil. (Reptilia-Tropiduridae). Studies on Neotropical FAúna and Environment 29(2):99-111.

VERRASTRO, L. \& KRAUSE, L. 1999. Ciclo reprodutivo de machos de Liolaemus occipitalis. Rev. Bras. Zool. 16(1):227-231.

VITT, L.J. \& BREITENBACH, G.L. 1993. Life histories and reproductive tactics among lizards in the genus Cnemidophorus (Sauria: Teiidae). In Biology of whiptail lizards (genus Cnemidophorus) (J.W. Wright \& L.J. Vitt, eds.). Oklahoma Museum of Natural History, Oklahoma, p. 211-243.

VITT, L.J. 1982. Reproductive tactics of Ameiva ameiva (Lacertilia: Teiidae) in a seasonally fluctuating tropical habitat. Can. J. Zool. 60:3113-3120.

VITT, L.J. 1983. Reproduction and sexual dimorphism in the tropical teiid lizard Cnemidophorus ocellifer. Copeia 2:359-366.

VITT, L.J. 1986. Reproductive tactics of sympatric gekkonid lizards with a comment on the evolutionary and ecological consequences of invariant clutch size. Copeia 3:773-786.

VITT, L.J. 1991. Ecology and life history of the wide-foraging lizard Kentropyx calcarata (Teiidae) in Amazonian Brazil. Can. J. Zool. 69:2791-2799.

VITT, L.J. 1992. Diversity of reproductive strategies among Brazilian lizards and snakes: the significance of lineage and adaptation. In Reproductive Biology of South American Vertebrates (W.C. Hamlett, ed.). SpringerVerlag, New York, p. 135-149. 


\section{ANEXO 1}

Anexo 1. Especimes Examinados

Annex1. Specimens Examined

\section{Cercosaura schreibersii}

Pontifícia Universidade Católica do Rio Grande do Sul (MCP) São Jerônimo: 14934 a 14939, 14958 a 14967, 15023 a 15028, 15090 a 15094, 15360 a 15364, 15436 a 15442, 15639 a 15644, 15777, 15778, 16006, 16743 a 17750, 17962, Don Feliciano: 15252 a 15318.

\section{Cnemidophorus lacertoides}

Pontifícia Universidade Católica do Rio Grande do Sul (MCP) São Jerônimo 14452 a 14458, 14724 a 14736, 14754, 14760,14832 a 14834,14881 a $14889,14901,14905,14913,14914,14917,14927$ a 14929,14945 a 14957,15006 a 15022,15081 a 15088,15323 a 15334, 15427 a 15435,15655 a 15667,15765 a 15775,15877 a 15888,15969 a 15977,16206 a 16220,16746 a 16750,16790 a 16795,17756 a 17768,17882 a 17885,17956 a 17958 